\title{
Biohybrid Walking Microrobot with Self-assembled Cardiomyocytes
}

\author{
Jinseok Kima, Eui-Sung Yoon a and Sukho Park ${ }^{b}$ \\ aNano-Bio Research Center, Korea Institute of Science and Technology \\ bShool of Mechanical Systems Engineering, Chonnam National University \\ Republic of Korea
}

\section{Introduction}

\subsection{Introduction to Microactuator}

Since the late 1980s, due to the rapid progress in MEMS (Micro Electro Mechanical System) technology, various types of mechanical structures, micro size sensors and actuators, could be fabricated, and technological progress in VLSI and SMT (Surface Mounting Technology) has contributed to the miniaturization of many electronic parts, which has led to the active development of microrobots. With the development of microrobots, research on the integration technology of micro actuators and micro sensors for microrobots has been in strong demand. Previously developed electric motors have advantages of over $90 \%$ high conversion efficiency and handling convenience. Miniaturized conventional actuators can be used as actuators of microrobot, but miniaturization rapidly decreases the drive force. Therefore, to move very small size equipment, a small actuator device based on a new actuation principle is needed [1].

Recently, MEMS micromachining of SMA (Shape Memory Alloy), Piezoelectric Ceramic Material and functional polymer material has been used to develop a new actuator. This new actuator has drawn attention as a suitable actuator for a microsystem. This new actuator can be miniaturized and made lighter, and especially, its generating power per weight ratio is much higher than those of conventional electromotive and hydraulic actuators. Therefore, it is expected to be suitable for miniature robots and microrobots [2].

Many other small and light actuators can produce much power. Because their structure materials and drive principles are diverse, their suitability of miniaturization must be evaluated, and their characteristics must be fully understood with careful consideration of the applications.

Electrostatic and electromagnetic motors are representative micro actuators. Because their generated powers are proportioned to the area of the electrode, they are more advantageous in miniaturization than previous actuators, whose powers are proportioned to the volume. 
Therefore, US-centered research has been actively developing micromotors, whose revolving part is smaller than the diameter of a hair strand by using silicon micromachining. Also, physical and chemical phenomena can be used as a micro actuator. For example, thermal expansion, piezoelectric effect, electric chemical reaction in addition to static electricity can be adopted as new actuation methods. Also, because each drive method has its own merits, the drive method that is suitable for meeting the actuation power requirements of the applied microsystem can be selected, so the most suitable design of the drive mechanism is needed. The basic requirements for micro actuators to be applied effectively to micro robots are as follows: compactness, light weight, high power density, high efficiency, good controllability, etc.

Polymers have many attractive characteristics; they are lightweight, inexpensive, fracture tolerant, and pliable. During the last twenty years, new polymers have emerged an additional important characteristic. This capability of the electroactive polymers (EAPs) has attracted much attention. Since they behave very similar to biological muscles, EAPs have acquired the moniker "artificial muscles." In the foreseeable future, robotic mechanisms actuated by EAPs will enable engineers to create devices previously imaginable only in science fiction [3].

Conventional microactuators use electrostatic, electromagnetic, pneumatic, piezoelectric, or thermal forces and require external power sources for operation. Although these actuators have been used in micro- and nano-scale systems, they have limitations in independent operation, such as in locomotion in a human digestive organ or migration in blood vessels. Unlike conventional actuators, cell-based actuators can use the glucose in physiological fluids as an energy source by converting glucose into ATP, and transforming this chemical energy into mechanical energy. Integrated systems like these cell-based actuators have been called hybrid systems, representing the material characteristics of micro- or nano-scale fabricated structures and biological components such as proteins and cells [4-5].

\begin{tabular}{|l|c|c|}
\hline \multicolumn{1}{|c|}{ Actuator type } & $\begin{array}{c}\text { Max. Pressure } \\
\text { (MPa) }\end{array}$ & $\begin{array}{c}\text { Max. } \\
\text { Efficiency (\%) }\end{array}$ \\
\hline Dielectric Elastomers & 16.2 & $60 \sim 80$ \\
\hline Electrostrict Polymer & 15 & - \\
\hline Electromagnetic (Voice coil) & 0.1 & $>90$ \\
\hline Piezoelectric & $110 \sim 131$ & $>90$ \\
\hline PVDF & 4.8 & - \\
\hline SMA & $>200$ & $<10$ \\
\hline $\begin{array}{l}\text { Mechano-chemical polymer } \\
\text { (Polyelectrolyte) }\end{array}$ & 0.3 & 30 \\
\hline $\begin{array}{l}\text { Natural Muscle } \\
\text { (Human skeleton) }\end{array}$ & 0.35 & $>35$ \\
\hline $\begin{array}{l}\text { Natural Muscle } \\
\text { (Mouse cardiac myocytes) }\end{array}$ & 0.00905 & - \\
\hline
\end{tabular}

Table 1. Comparison with the capabilities of various microactuators [3] 
Natural muscles are considered highly optimized systems since they are fundamentally the same for all animals and the difference between species are small. The operation of muscles depends on chemically driven reversible hydrogen bonding between two polymers, actin and myosin. The reason why this paper chose the cardiac muscle of rat as an actuator for microrobot is that the cardiac muscle does not need external stimulation.

\subsection{Muscle Powered Microrobot}

Biomimetic refers to human-made processes, materials, devices, or systems that imitate nature. Billions of years of "natural R\&D" have resulted in effective, optimized biological solutions that really work. By studying and mimicking nature's processes and structures, scientists and engineers can develop nature inspired solutions that are far more effective than solutions conceived and developed exclusively by man. Biomimetic artificial machines built with hybrid components (materials partly synthetic and partly biological in origin) offer the opportunity to combine enhanced sensitivity with robustness and the possibility to extend their application to diverse environmental conditions [6]. As examples of micro/nano machine using hybridization between organic and inorganic components, researches on adenosine triphosphate (ATP) biomolecular motors $[7,8]$, a microorganism carrier in microchannel [9], a walking microdevice driven by micromuscle [10,11], and a pump actuated by cultured cardiomyocytes [12] were reported. Although, bimolecular motors are interesting, they can generate only between $5-60 \mathrm{pN}$ forces [13] and are not robust to actuate microstructures. Micromuscles can be an alternative to microactuator for a micro-sized biomimetic system. Previous studies using cell based actuators [11-12] have shown very attractive results, but for advanced biomimetic systems, understanding and exploiting higher-order assemblies for micromuscles are key points of today's quest [6]. Structure and functional changes ensue in cardiac cell networks when cells are guided by three-dimensional scaffold topography, such as enhanced actin cytoskeleton organization, higher nuclear eccentricity [14] and altering gene expression, protein localization [15-16], cell signaling [17] and the intracellular calcium dynamics [18]. These topology-induced changes are expected to enhance the mechanical activity of cells [14]. However, until now, there has been no suitable tool to validate this enhancement.

We propose a biomimetic micromachine made of a silicone elastomer polydimethylsiloxane (PDMS) and self organized cardiomyocytes, which allows free motions in physiological liquids based on the increase of cell power in a 3D microenvironment. The fabrication method and the formation and function of the legs in the machines mimic the bottom-up process of nature, the patterns in jelly fish and the functions of a real heart. Fig.1 shows the microrobot structure mimicking the legs of a jelly fish.

To make it move like the synchronized motion of a jellyfish without external power supply, we primarily cultured the heart cells of a mouse on the microrobot device and used the beating motion of the heart cells as a power source. Also, to mimic the muscle bundle structure on the legs of a jellyfish, we made in vivo-like grooved surfaces. From this, we could obtain motions of the machine driven by the contractility of 3D controlled cardiomyocytes. We found the obvious increase of mechanical activity of cardiomyocytes in the grooved surfaces compared with that in flat surfaces by using culturing cardiomyocytes on flat and grooved PDMS microcantilevers. We can suggest the increase of force due to the 
arrangement and increment of the cells in the same area. The process and materials used in this study is noninvasive to cells, and have the potential of high throughput of the microdevice.
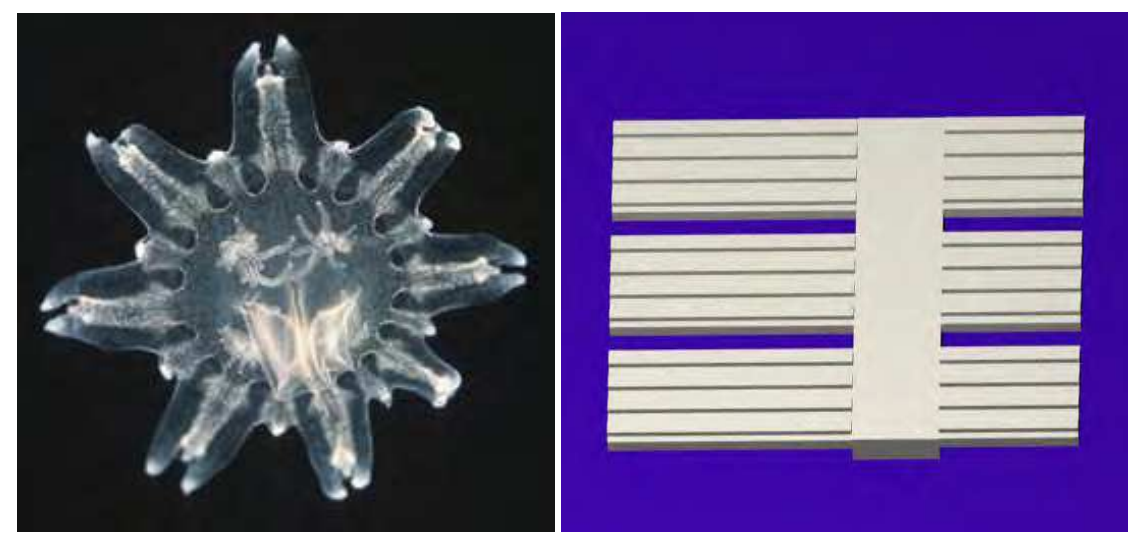

Fig. 1. A. Concept design to mimic the Ephyra of the moon jelly fish. Muscle bundles in middle of legs are used for movement. B. Mimicked polymer based backbone structure of a microrobot

\section{Biopolymer Hybrid Devices}

\subsection{Design of biopolymer actuator \& sensor}

\subsubsection{Flat surface cantilever}

In this study, a microcantilever was designed and fabricated, using both synthetic and natural materials. Living cells were cultured on a cantilever made of flexible transparent PDMS elastomer. Therefore, the proposed cantilever can be considered as a hybrid biopolymer cantilever. PDMS has a low Young's modulus, which can be tuned from 360 to $868 \mathrm{kPa}$ by controlling the ratios of its components [19], and thus is extremely sensitive to external stress and can be used to achieve large deflections. PDMS is also inexpensive, optically transparent and suitable for use with optical detection methods. Additionally, it is compatible with uses in biological studies because it is impermeable to water, nontoxic to cells, and permeable to gases. Finally, it can be easily fabricated and bonded to other surfaces [20]. Culturing primary cardiomyocytes on the microcantilever leads to selforganization of the cells on the structure, giving a parallel arrangement of cells in the hybrid system. The system can thus avoid problems arising from inefficient dissection and attachment of the muscle tissue to the microsystem by hand. Since the presented system was realized with simple micromolding a technique using PDMS, a large quantity of samples can be produced cheaply and easily. Our method is also safe and noninvasive, because the entire device is biocompatible and there are no hard contacts or structural constraints that can affect myocytes function or morphology. The important feature in our system over previous contractile force measurement techniques is that our system can quantitatively measure contractile forces on a specific microsized area in real time, and this capability should open opportunities for better understanding of the mechanisms of heart failure and 
promote further design of optimal microscale hybrid biopolymer actuators and microdevices.

\subsubsection{Cell culture}

The cardiomyocytes on the microcantilevers were obtained by aseptically isolating the heart of a neonatal Sprague-Dawley rat at day 1 and briefly washing it with Hank's balanced salt solution (Gibco Invitrogen Co., Grand Island, NY). After removing the ventricles, the remaining tissues were minced and incubated in a $0.3 \mathrm{mg} / \mathrm{mL}$ collagenase solution containing $0.6 \mathrm{mg} / \mathrm{mL}$ pancretin (Sigma Chemical Co.). The isolated cardiomyocytes were seeded directly onto the hybrid biopolymer cantilever at a cell density of 5_103 cells/mm2 and cultured in Dulbecco's modified Eagles' medium (Gibco Invitrogen) containing 10\% fetal bovine serum (Sigma), $50 \mathrm{mg} / \mathrm{mL}$ streptomycin, and $50 \mathrm{mg} / \mathrm{mL}$ penicillin (Gibco Invitrogen) at $37 \mathrm{C}$ in air with 5\% CO2. The cell activation level reached its maximum after 72-96 $\mathrm{h}$ of culture. The motion of the microcantilevers was measured at the 96th hour by using two microscopes, which monitored lateral and vertical motions, as described in Park et al. (2005) [21].

\subsubsection{Molding master fabrication}

By using a flexible and thin PDMS matrix layer, we intended to maximize the bending displacement of thin PDMS by generating a force through the arrangement of contractile cardiomyocytes. The flexible and thin PDMS matrix layer was fabricated by the sandwich molding process [22], as shown in Fig. 2. The sandwich molding process is a useful technique and was used to create a PDMS 3D microrobot structure with an ideal thickness of , $20 \mathrm{~mm}$ by pouring PDMS between a bottom Si wafer master and a top glass wafer master and then compressing the layers. The bottom master was fabricated on the Si wafer using a deep silicon etching process and a thick negative photoresist (PR) (SU-8). First, grooves were patterned onto a new silicon wafer using an AZ1512 PR. The Si wafer was then deeply etched to fabricate a grooved surface, as shown in Fig. 1(a). After removing the AZ1512 PR, the Si wafer was coated with SU-8 to develop the legs of the microrobot, as shown in Fig. 1(b). The microrobot legs were fabricated from the bottom Si master so that the grooved shape would be on the leg surface. The microrobot body was fabricated from the top glass master. After coating the glass wafer with a $\mathrm{Cr} / \mathrm{Au}$ layer, we patterned the microrobot body using the AZ1512 PR, as shown in Fig. 1(c). Finally, the top master fabrication was completed by removing the $\mathrm{Cr} / \mathrm{Au}$ layer from the etched glass wafer, as shown in Fig. 1(d).

\subsubsection{Polymer device fabrication}

Several previous reports have investigated the fabrication of $3 \mathrm{D}$ structures with polydimethylsiloxane (PDMS). The most common molding method is the sandwich molding technique using a single molding master [22, 23] In this method, the master, another flat wafer, and the PDMS are all stacked together to produce the 3D PDMS structures. Unfortunately, this method only allows specific geometries to be created on one side. Although a method for fabricating microstructures on both sides has been developed, it does not easily yield thin micromembranes and is difficult to use for wafer-level fabrication since the PDMS used as the top master is too flexible for high-pressure applications and may shrink during curing, particularly over areas greater than $1 \mathrm{~cm}^{2}$. To 
overcome these problems, we propose a 3D molding aligner system that can align the two mold masters and stack them under high pressure to fabricate a 3D structure with micromembranes at the wafer level. To facilitate the detachment of the PDMS structures, the top and bottom masters were coated by plasma polymerization of $\mathrm{C}_{4} \mathrm{~F}_{8}$ using inductively coupled plasma-reactive ion etcher (ICPRIE). The PDMS mixture was poured onto the bottom master, as shown in Fig. 1(e). After the top master was placed on the PDMS mixture, the stack, comprised of the bottom master, the PDMS, and the top master, was placed between two aluminum plates. To align the two masters using the $3 \mathrm{D}$ mold aligner, the geometries of the two masters were observed through the windows at the top of the wafer chuck. The two masters could be aligned to a precision of less than $2 \mathrm{~mm}$ using an optical microscope. Following the alignment, the stacked bottom master, PDMS, and top master were clamped, as shown in Fig. 1(f). The clamped stack was cured in an oven for $2 \mathrm{~h}$ at 100 ${ }^{\circ} \mathrm{C}$. After curing, a thin PDMS replica was peeled from the master, as shown in Fig. 1(g).

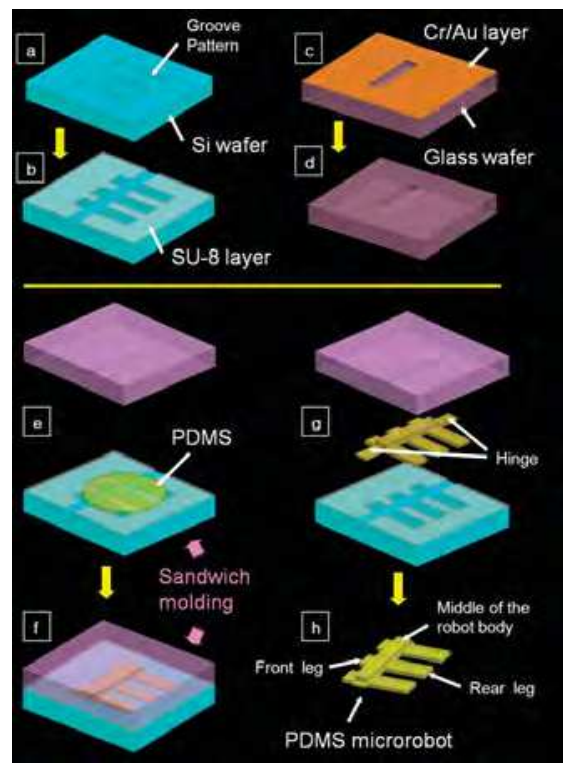

Fig. 2. Fabrication process of the PDMS microrobot. (a) Etched groove pattern on the bottom Si master. (b) Coated and patterned SU-8 layer on the bottom Si master. (c) Patterned Cr/ Au layer on the top glass master. (d) Etched pattern on the top glass master. (e) Poured PDMS between the top and bottom masters. (f) Clamped top and bottom masters. (g) Detaching the molded PDMS

Figure 3 shows the concept of the process for making a hybrid device by primarily culturing neonatal rat heart cells on a micro device made with PDMS polymer. As reason for making a hybrid device and its working principle were previously explained, and the detailed fabrication method and process will be directly explained in this chapter. 


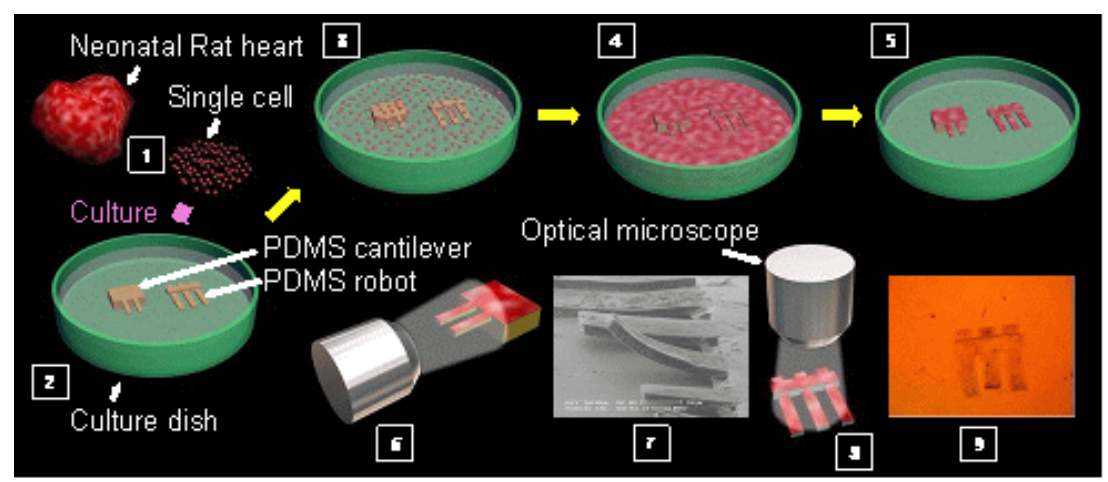

Fig. 3. Full preparation process of microrobot to observe the motion(1) Extract heart single cell from neonatal rat heart, (2) Prepared PDMS structure, whose surface will culture the cardiomyocytes, (3) Primary cardiomyocytes culture on the culture dish including PDMS structure, (4) Incubating cultured cardiomyocytes, (5) Detach PDMS structure from the original culture dish and move to a new culture dish to observe the movement of the PDMS structure, (6) Schematic image to observe the vertical movement, (7) Microscopic image of the vertical view, (8) Schematic image to observe the lateral movement, (9) Microscopic image of the lateral movement.

\subsubsection{Engineered surface cantilever to concentrate the contraction force}

A heart attack is the most dreaded heart disease. And chronic heart attacks, even slow-going, are the most common and fatal. Heart muscles weaken slowly and its cells simply can't contract. In this case, each heart muscle cell has longer pulsation and loses its force. Therefore, the information on the contractile force of the heart cell is expected to play an important role in finding the basic remedy and substitution for cells with heart disease [24]. Although force measurements at the cellular tissue and whole organ levels have been performed [25-26], influence from outside factors cannot be entirely excluded, giving rise to inaccurate measurements of the force of heart cells. Therefore, there have been several approaches proposed and methods developed to measure the contractile force of cardiomyocytes either directly or indirectly.

In the first approach, micro fabrication technology was used to make a force transducer that would reduce the size of the device for measuring the contractile force of cardiomyocytes [27]. In this system, two micro clamps hold each end of a cardiomyocyte cell to measure the contractile force of the cell. However, this approach involves cell manipulations, which may have unknown effects on the cells and their functions.

In the second approach, an array of micro-scale elastic posts was developed. The attached cells bend each post independently because the forces of a cell for adhesion were used locally. Poly-(dimethylsiloxane) (PDMS) pillar arrays were used as elastic posts [28]. However, the contact between the cell and the heads of pillars may have some effects on cell membranes and cardiomyocytes functions. Moreover, the spreading and morphology of the cell on the flat surface and on the pillar show different aspects, which seems to have a great influence on the contractile force of the cardiomyocytes cell.

The difference of the force of cardiomyocyte cells due to the surface change of the cantilevers was measured. Cantilevers are often used in MEMS devices, not only as 
actuators but also as sensors. The microcantilever can be used as a sensitive chemical/biological sensor through mechanical methods, by detecting the deflection of the cantilever due to surface stress. PDMS was used as the material of a biopolymer cantilever. PDMS is inexpensive, optically transparent, and suitable for use with optical detection methods. Also, it can be easily made and bonded to other surfaces [29].

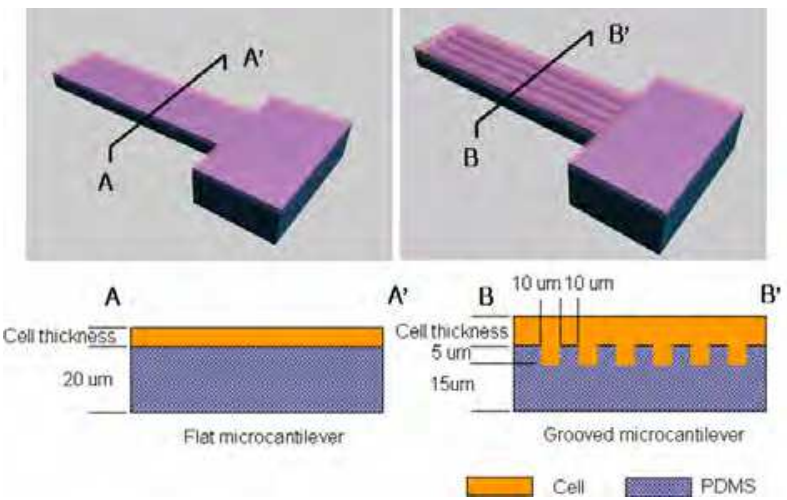

Fig. 4. Schematics of a cross-section of a 3D hybrid biopolymer microcantilever structure: left is a flat microcantilever and right is a grooved microcantilever.

The hybrid biopolymer microcantilever array consisted of five different sizes of microcantilevers, which were 50,100,150, 200, and 300 $\mu \mathrm{m}$ wide and five times the widths in length, respectively. However, all cantilevers were $20 \mu \mathrm{m}$ thick. It was found that cantilevers longer than $1 \mathrm{~mm}$ frequently stuck onto the substrate or were bent, probably due to both the flexibility of the PDMS microcantilever and the cell mass formed by the self organizing cells. Therefore, as mentioned, all data obtained from cantilevers longer than $1 \mathrm{~mm}$ were eliminated. As the difference occurred in thickness in a grooved cantilever unlike the surface flat cantilever shown in Figure 4, the structural deflection change ratio was verified on the same force before culturing the cardiomyocytes cell.

\begin{tabular}{|l|l|l|l|l|l|l|l|l|}
\hline & S1 & S2 & S3 & S4 & S5 & S6 & Ave. & S.D. \\
\hline $\begin{array}{l}\text { Displacement increment } \\
\text { percentage of the grooved } \\
\text { cantilever (\%) }\end{array}$ & 25.8 & 24.2 & 39.5 & 39.2 & 35.6 & 25.7 & 31.7 & 7.2 \\
\hline $\begin{array}{l}\text { Contractile force increment } \\
\text { percentage of the grooved } \\
\text { cantilever (\%) (analytical solution) }\end{array}$ & 69.5 & 67.4 & 88.0 & 87.7 & 82.8 & 69.4 & 77.5 & 8.9 \\
\hline $\begin{array}{l}\text { Contractile force increment } \\
\text { percentage of the grooved } \\
\text { cantilever (\%) (FEM) }\end{array}$ & 67.3 & 64.9 & 85.3 & 84.9 & 80.1 & 66.9 & 74.8 & 8.7 \\
\hline
\end{tabular}

Table 2. Increments of the displacement and contractile force of the cardiomyocytes on the grooved microcantilever

A grooved cantilever was expected to bend much more than a surface flat cantilever upon a small force structurally, as shown in Figure 4. As expected, from the result of the simulation, 
it was confirmed that a grooved cantilever could bend more than a surface flat cantilever as shown in Table 2. Based on measured displacement and analytical calculation or FEM modeling, shear forces on the microcantilevers were calculated. Table 1 shows the increments of the displacement and contractile force of the cardiomyocytes on the grooved microcantilever compared with those of the flat microcantilever. To compare the contractile forces of cardiomyocytes on the grooved microcantilever with that on the flat one, the structural difference of two microcantilevers had to be considered.

Synthetically considering the above two factors, to yield the same amount of bending displacement by the two types of microcantilevers, the grooved microcantilever needed $34.8 \%$ higher contractile force than that of the flat microcantilever. Similarly, according to FEM analysis, the flat microcantilever yielded $32.8 \%$ more bending displacement to the same value of shear force than the grooved microcantilever. Nevertheless, the cardiomyocytes cultivated on the grooved microcantilever gave a $25-40 \%$ increase in bending displacement from the percentage increase in bending displacment on the flat microcantilever. The contractile force of the aligned cardiomyocytes on the grooved cantilever yielded a $67-88 \%$ increase in displacement, which is higher than the percentage increase of displacement of the cardiomyocyte on the flat cantilever. The result of FEM analysis, $65-85 \%$ increase in displacement, was similar to the analytical solution above. The contractility of the cardiomyocytes on the grooved microcantilever was much higher than that on the flat microcantilever. Previously, many researchers developed microtechnology methods to measure the contractile force of cardiomyocytes (Lin et al., 2000; Tan et al., 2003; Zhao and Zhang, 2005; Balaban et al., 2001). The stress was found to vary between 2 and 5 $\mathrm{nN} / \mathrm{mm}^{2}$. The variation of stress from the cardiomyocytes, reported in the range from 2 to 5 $\mathrm{nN} / \mathrm{mm}^{2}$, was verified using our flat microcantilever (Park et al., 2005). Also, in this paper, it was identified that the stress variation of cardiomyocytes concentrated on the grooved surface increased $4-10 \mathrm{nN} / \mathrm{mm} 2$ and the average contraction increased $65-85 \%$.

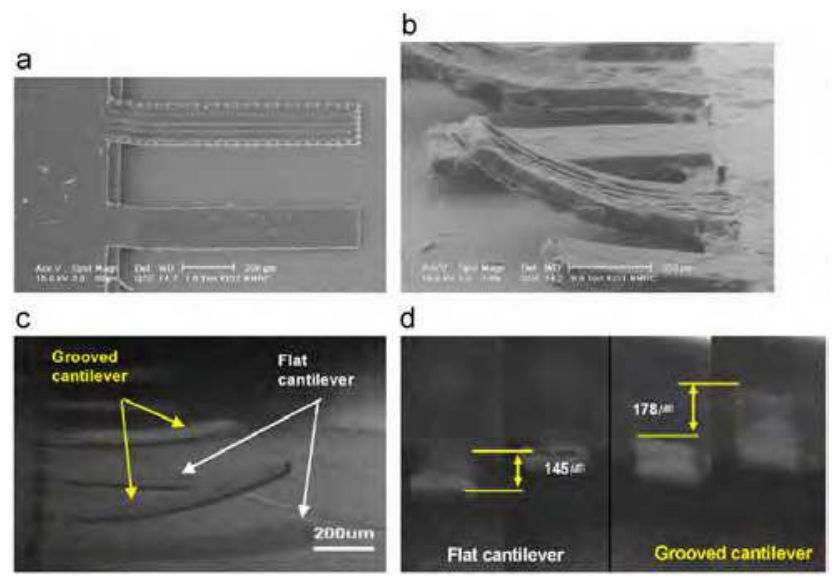

Fig. 5. Images of the flat and grooved microcantilevers: (a) Fabricated PDMS flat and grooved microcantilever, (b) ESEM image of the hybrid organic-inorganic flat and grooved microcantilevers, (c) and (d) still images from video recordings of the vertical motion of the 200x1000 m hybrid biopolymer microcantilevers. 
Figure 5(a) and (b) show the fabricated PDMS microcantilevers and cultured cardiomyocytes on the flat and grooved microcantilevers. After the cardiomyocytes were cultured, the initial deflection of the grooved microcantilevers became larger than that of the flat microcantilevers. Compared with the actin filament of the cardiomyocytes on the flat microcantilever, that on the grooved microcantilever was well organized with a higher order. Figure 5 (c) and (d) show still images from video recordings of the vertical motion of the 200x1000 m hybrid biopolymer microcantilevers. The vertical motion of the hybrid biopolymer microcantilevers clearly showed the difference of the bending displacement between the flat and grooved microcantilevers. With respect to the deflections, the displacement of the grooved microcantilevers was larger than that of the flat microcantilevers. Analytical calculation and FEM analysis with ANSYS (ANSYS, Inc.) were performed to quantify the contractility of cardiomyocytes based on these experimental results. For a more realistic simulation, cardiomyocytes was modeled as a material contracting in the longitudinal direction in contact with the PDMS structure, and thus the contractile force was modeled as a shear force exerted at the interfacial area between the cardiomyocytes and PDMS due to the longitudinal contraction of the cardiomyocytes. Young's modulus and Poisson's ratio of the PDMS and cardiomyocytes were assumed to be $750 \mathrm{kPa}$ and 0.49 [20], and $40 \mathrm{kPa}$ [30] and 0.49 [31], respectively. Information on the height of the cells is essential for calibrating the focal pressure of the cardiomyocytes at an interface. In general, the thickness of the cells differs according to surface conditions. When cells are cultured on a flat surface, their height is approximately $5 \mathrm{~mm}[16,21]$. However, the height of the cells on a grooved surface is approximately $10 \mathrm{~mm}$ [16].

\section{Hybrid Biopolymer Microrobot}

\subsection{Introduction of biopolymer microrobot}

A proposed micromachine was made of silicone elastomer polydimethylsiloxane (PDMS) and self organized cardiomyocytes, which allow free motions in physiological liquids based on the increase of cell power in a 3D microenvironment. In order to fabricate a 3D environment in a robot body, specially designed 3D molding aligner was invented [32].

\subsection{Fabrication}

\subsubsection{Fabrication of molding master for 3D PDMS microrobot}

As the same fabrication method of microcantilever, the sandwich molding process was used to fabricate a 3D PDMS microrobot. Figure 6 illustrates fabrication results from the precise alignment-based sandwich micromolding process. The silicon master with two level photoresist structures (Figure 6 (a)) and the glass master (Figure 6 (b)) were fabricated successfully. Figure 6 (c) shows the instant of alignment of the top and bottom masters carried out by our 3D micromolding aligner. A fabricated complex 3D microstructure with top and bottom geometries is shown in Figure 6 (d) as an example. 


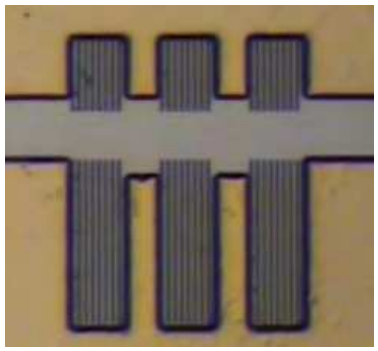

(a)

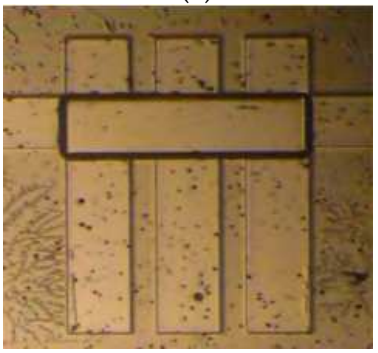

(c)

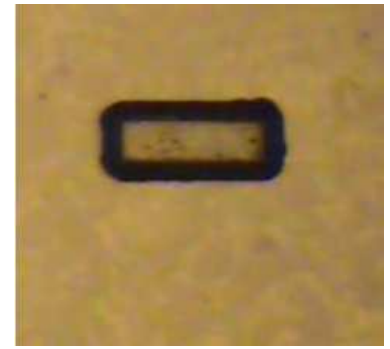

(b)

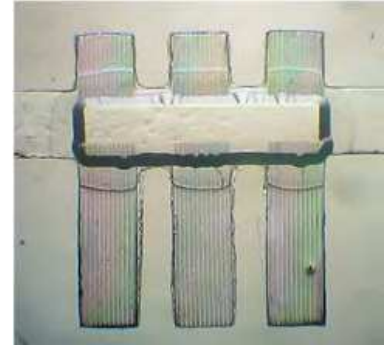

(d)

Fig. 6. Microrobot fabrication results using the sandwich micromolding process with precise alignment (a) Bottom silicon master. (b) Top glass master. (c) Alignment of top and bottom masters via a 3D-micromolding aligner. (d) the fabricated complex 3D PDMS structure.

To primary culture cardiomyocytes on the manufactured microrobot device through the PDMS molding process, a pre-process is needed. First, the PDMS device detached from the molding master is washed in $70 \%$ ethanol to remove impure particles. It is then immersed in $70 \%$ ethanol for an hour for sterilization. Then it is taken out, and dried for 30 minutes on the clean bench under UV light. The fresh PDMS surface is in hydrophobic condition and this prevents the adhesion of proteins and cells. Therefore, $\mathrm{O}_{2}$ plasma treatment was applied to increase adhesion forces between the PDMS surface and extracellular matrix. PDMS consists of repeating - $\mathrm{OSi}\left(\mathrm{CH}_{3}\right)_{2}$ - chains. The chain of $\mathrm{CH}_{3}$ groups makes the surface of the PDMS hydrophobic. The surface can be changed to hydrophilic by exposure to $\mathrm{O}_{2}$ plasma for 5 min. Plasma enhances cell adhesion onto the PDMS by oxidizing the surface of the PDMS to silanol $(\mathrm{Si}-\mathrm{OH})[20,69]$. Then, the device is coated by immersing it in the ECM (Extra Cellular Matrix: fibronectin) solution overnight and taken out. Then, it is dried on the clean bench under UV light for an hour. Then the device to culture the cell primarily is ready, as shown in Figure 20 (2).

Then the cell is seeded on the device and 5 days later, the device is detached at the bottom and cut the hinge on the device. Then, the robot device can move freely. Just before observing through an optical microscope, turn over the device by pipetting and look at the walking motion of the microrobot. 


\subsection{Experiment}

\subsubsection{Monitoring of Walking Motion of Microrobot}

To observe the vertical motion of the biomimetic microstructure, a CCD camera microscope (CCD CAM scope, SomeTech Vision) was used. Then, the motion of a biomimetic micromachine was measured using an inverted microscope (Olympus IX 81, Olympus) and captured by a digital camcorder (DCR-PC350, Sony Corp., Japan), as shown in Figure 20 (8). The captured movies were transferred into digital movie files at 30 frames/sec, and then we analyzed the walking motion of the biomimetic micromachine using sequential video frames.

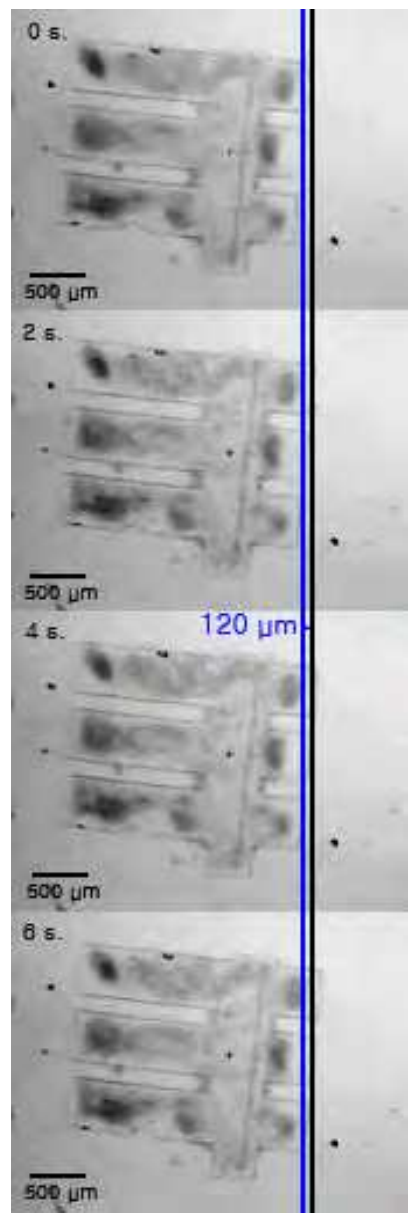

(a)

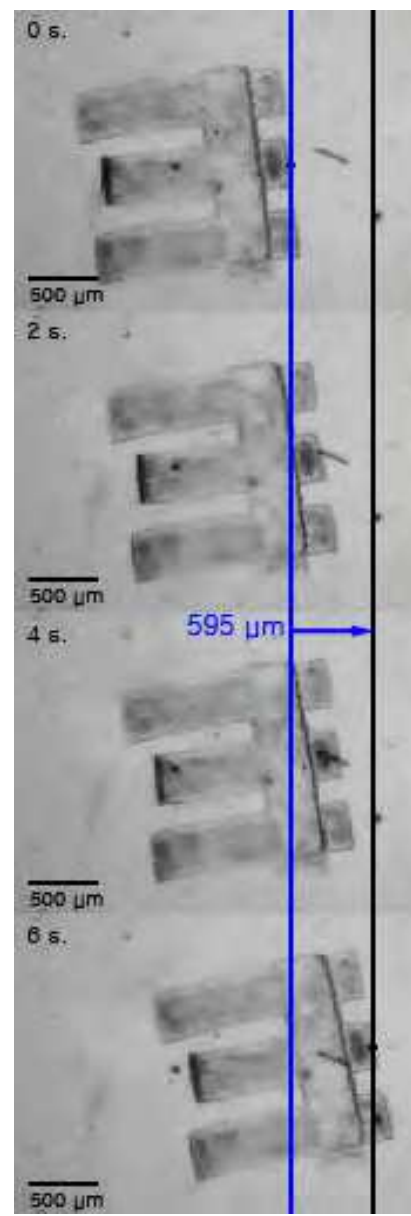

(b)

Fig. 7. Sequential images for the movement of biomimetic micromachine (a) flat surface cantilever type legs, (b) groove surface cantilever type legs

The biomimetic micromachine is designed and fabricated to achieve walking movement. The micromachine has asymmetric structure, which has 3 front and 3 rear legs of different 
length. To mimic the legs of the ephyra and to realize a stable and simple structure, multiple legs are considered and all the legs are connected to the middle of the robot body to synchronize with the cardiomyocytes. For the forward movement of the microrobot, the legs are aligned in the horizontal direction and the front legs are made to be shorter than the rear legs. To concentrate the cardiomyocyte cell force, groove patterns are engraved on the surface of the legs. After about 72 to $96 \mathrm{~h}$ of culture, cardiomyocytes began to beat synchronously and all legs shows vertical displacement owing to the contractile force of the cardiomyocytes. Finally, the biomimetic micromachine activated by the contractile force of the cardiomyocytes begins to move, as shown in in Figure 7 . Figure 7 (a) and (b) show the movement of the micromachine with flat surface cantilever type legs and groove surface ones, respectively. In case of the micromachine with the flat surface cantilever type legs, the cardiomyocytes on the surface on legs show synchronous beating, but the microrobot appears to show only a small advancement. However, the micromachine with groove surface shows forward movement. The average beating frequency was $1.4 \mathrm{~Hz}$ and its average step stroke was $100 \mu \mathrm{m}$, and thus, the calculated speed was about $140 \mu \mathrm{m} \cdot \mathrm{s}^{-1}$.

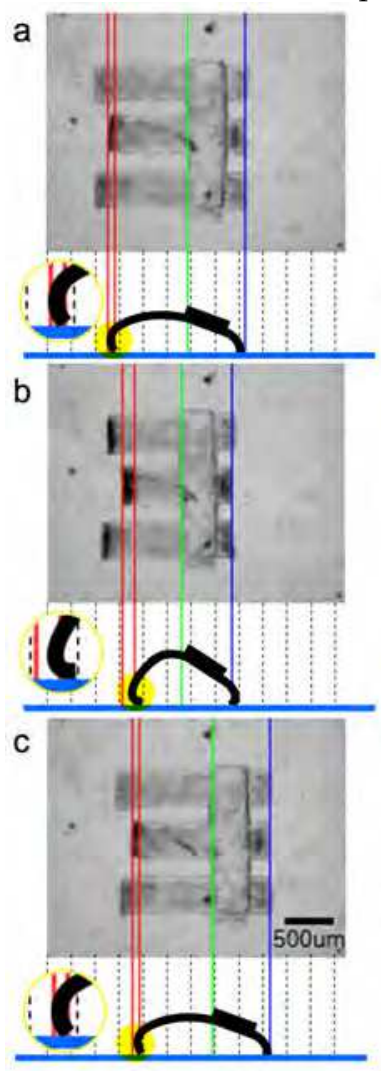

Fig. 8. Still images showing the sequential movement of the micromachine during one step (a) Before contraction of the leg (b) During contraction of the leg (c) After relaxation of the leg. Blue, green and red lines represent the edges of the vertical image of the micromachine at (a), (b) and (c) state, respectively. 
However, the average speed of the biohybrid micromachine was measured to be about $125 \mu \mathrm{m} \cdot \mathrm{s}^{-1}$. The difference between the calculated speed and the average speed will be explained with respect to the walking mechanism of the micromachine.

For forward movement, it is important that friction, related to the surface area of contact, be adjusted so that the microdevices can maximally use its muscle power [11]. The proposed micromachine has 3 front legs and 3 rear legs of different lengths. By the contractile force of the cardiomyocytes, cultured on the micromachine, the deflection of the rear legs of longer length is much larger than that of the front legs. At the end of the legs in contact with the substrate, the asymmetric design of the micromachine produces the difference of the surface area of contact and thus causes an imbalance of the friction force between the legs and the substrate. Therefore, the micromachine can move in the direction of the front legs of shorter length. The sequential video images of Figure 8 show the walking motion of the biomimetic micromachine during one step.

\section{Measurement and Discussion}

\subsection{Long-term Monitoring of Microrobot}

\subsubsection{Experimental setup}

In a hybrid device, the compatibility between the organic and inorganic materials is the most important parameter. Therefore, the cells on the structure were monitored for a long time and checked to determine if the proposed method was biocompatible and feasible. Although the cardiomyocytes started beating when they started to aggregate, but they was monitored from the 5 th day when force was gathered by their synchronization.

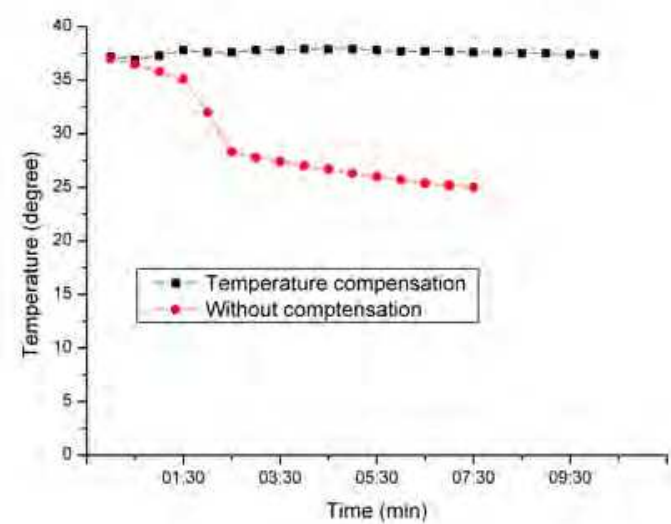

Fig. 9. Comparison of temperature variation between temperature compensation and temperature without compensation

To observe the hybrid device, the temperature change of the solution in the dish taken out from the incubator oven was checked. The graph in figure 9 shows the temperature change of the culture solution in the Petri dish filled with the cardiomyocytes when the cardiomyocyte cultured Petri dish was taken out from an incubator oven, and the temperature change was checked with an inverted microscope. When there is no 
temperature compensation, the inner temperature of the bioFAB is $25^{\circ}$. Because of the sudden drop in temperature, cells seemed to have been damaged. Therefore, temperature compensation was tried using a light bulb, and an experiment condition which could conserve the constant temperature was set up.

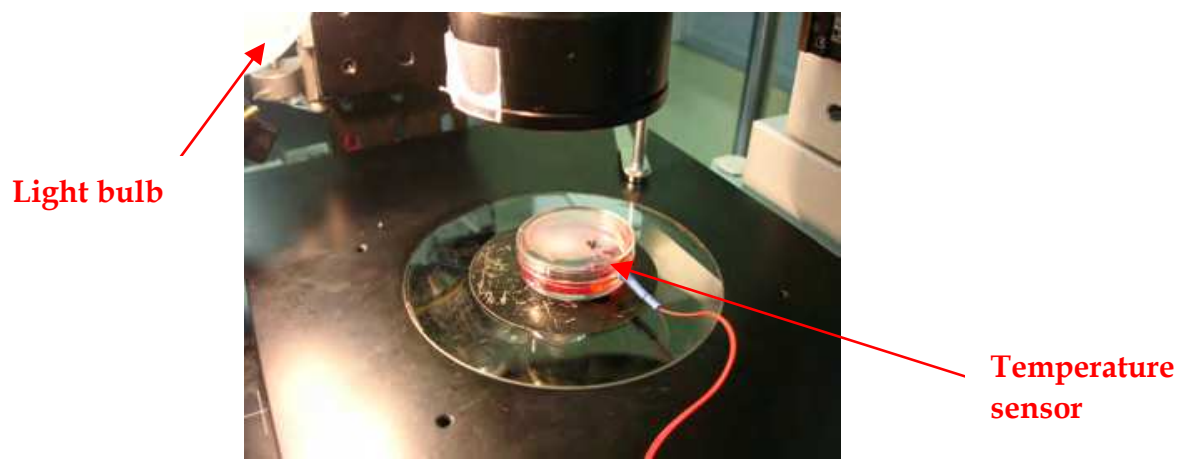

Fig. 10. Experimental setup to compensate the temperature

Figure 10 shows the experimental setup of temperature compensation using a light bulb and the setup measuring temperature by inserting a sensor in the Petri dish. The AD converted value of the voltage corresponding to the change of the resistance value in the thermostat was converted to temperature by interfacing.

\subsection{Frequency and Displacement vs. Force}

\subsubsection{Frequency vs. Bending displacement}

The beating frequency and the displacement of the structure due to the contractile force of the cardiomyocytes were measured from the 5th day. As shown in Figure 11 (a), frequency became slower with time. Bending displacement increased, and on the 10th day, it was biggest, as shown in Figure 11 (b).

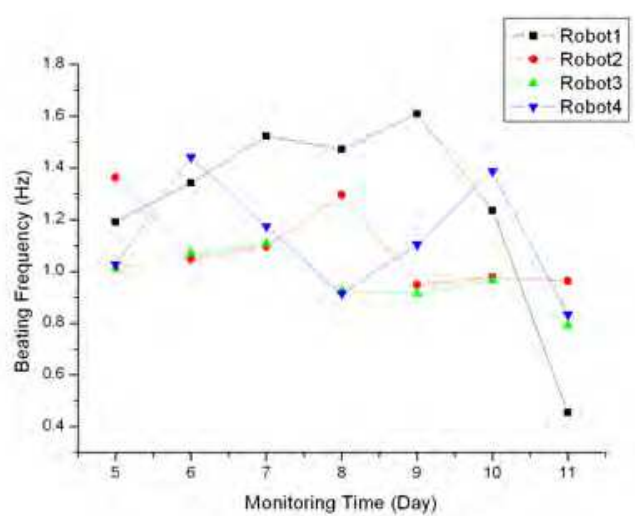

(a) 


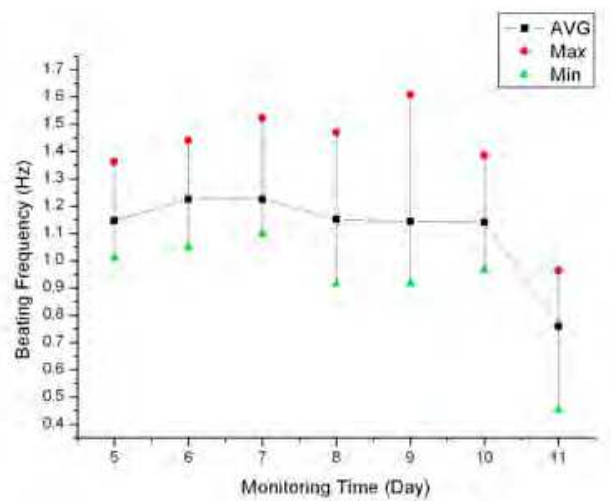

(b)

Fig. 11. Long-term monitoring results of the cardiomyocytes activity on the polymer structure (a) Beating frequency of the cardiomyocytes according to the number of days (b) Average frequency of the cardiomyocytes according to the number of days

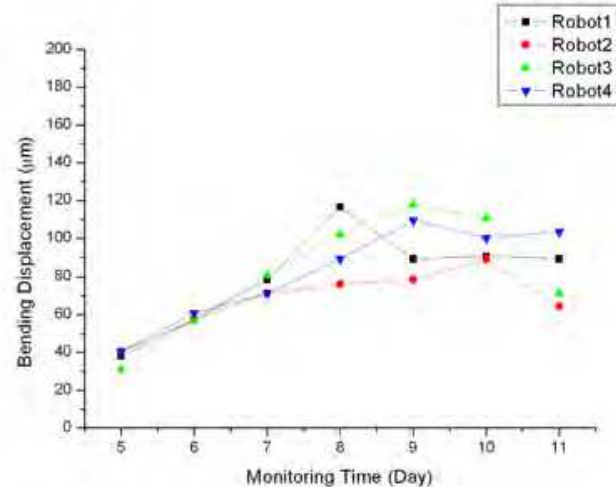

(a)

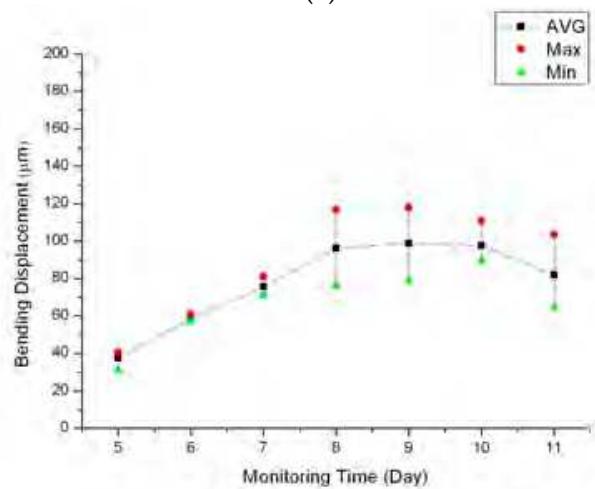

(b)

Fig. 12. Long-term monitoring results of the cardiomyocytes activity on the polymer structure (a) Step increment of the microrobot legs according to the number of days (b) Average step increment of the microrobot legs according to the number of days 
Actually, the whole distance walked by the walking robot could not be measured, but when you multiply the frequency per day by the step increment, you can obtain the velocity per day. And when you multiply this result by time again, you can obtain the movement distance per day. So, by calculation, the total movement distance of the micro robot for 7 days is expected to be more than 50 meters.

As shown in the above experimental result, cardiomyocytes primary cultured on the polymer fabricated structure was checked to see if they were beating normally and operating well as an actuator. From this, it was found that PDMS used as the backbone of the hybrid device was biocompatible, and that the plasma treatment and extra cellular matrix coating used to increase the adhesion with cells made a stable and healthy environment for the cells to live. Therefore, a long-term actuator was found to be successful because it operated for over 10 days as an organic/inorganic hybrid actuator. Even though the beating frequency became slower, contractile force became stronger. Also, the microrobot constantly moved for 7 days and showed that it could move an incredibly long distance.

\section{Conclusion}

By growing rat muscle tissues on a polymer backbone, we demonstrated the movement of the cell powered microrobot. From the viewpoint of a walking microrobot driven by muscle, the cell powered microrobot has novel originality and great significance in the robotics field. The proposed microrobot has the following characteristics: (1) the self-assembled hybrid microrobot consists of biotic muscle cells and PDMS backbone, which is well-known as a biocompatible material. (2) The surface of PDMS backbone is engineered into a groove pattern, which promotes higher order cell concentration and realizes higher generative force of the muscle cells than a 2D culture surface. (3) PDMS is easily fabricated by the? a? micromolding procedure and thus high throughput and mass-production of the cell powered microrobot become possible. (4) The long term monitoring result of the primary cultured cardiomyocytes on engineered surfaces is firstly reported and analyzed. Consequently, the walking robot showed reliable and long-term actuation performances. During the organization of the cardiomyocyte-PDMS hybrid microrobot, the groove surface provided an adverse environment in the initial stages of cell attachment and at adaptation to the surfaces by the modifications in the specific gene expression of the isoforms of troponin and connexin. However, once the cells settled down, more vigorous and synchronous propelling forces were generated through the tighter cell-cell contacts, intracellular arrangements of vesicles and cytoskeltons and higher stimuli according to the microenvironment on the groove surfaces.

As quantitative results, the stress of $2-5 \mathrm{nN} / \mu \mathrm{m}^{2}$ was calculated, which was induced on the flat surface of the microcantilever by the contractile force of the cardiomyocytes. Then, the force of the cardiomyocytes cultured on the groove surface microcantilever increased up to $60 \%$ higher than that on the flat surface cantilever. Finally, the forward movement of the micro-robot with the groove surface was monitored, and the measured speed was about 140 $\mu \mathrm{m} \cdot \mathrm{s}^{-1}$. 


\section{References}

[1] Hiroyuki Fujita, (1998). Microactuators and micromachines, Proceedings of the IEEE, v.86 no. 8, pp. 1721-1732

[2] Friedrich, C.R.; Fang, J.; Warrington, R.O. (1997). Micromechatronics and the miniaturization of structures, devices, and systems, IEEE transactions on components, packaging, and manufacturing technology Part C, Manufacturing, v. 20 no. 1, pp. 31-38

[3] Bar-Cohen, Y., (2006). Biomimetics: biologically inspired technologies, Second Edition (SPIE Press Monograph) Vol. PM136

[4] Ho, D.; Chu, B.; Schmidt, J.J.; Brooks, E.K.; Montemagno, C.D. (2004). Hybrid proteinpolymer biomimetic membranes, IEEE Trans. Nanotech. 3, pp. 256-263

[5] Neves, H.P.; Schmidt, J.J.; Soong, R.K.; Montemagno, C.D., (2002). Hybrid micro-and nanobiomechanical systems: a control perspective, World Automation Congr. Proc. 5th Biannual, pp 275-279

[6] Spatz, J. P. (2005). Bio-MEMS: Building up micromuscles, Nature Mater. 4, pp. 115-116

[7] Soong, R. K.; Bachand, G. D.; Neves, H. P.; Olkhovets, A.G.; Graighead, H. G.; and Montemagno, C. D. (2000). Powering an inorganic nanodevice with a biomolecular motor, Science 290, pp. 1555-1558

[8] Rondelez, Y.; Tresset, G.; Nakashima, T.; Kato-Yamada, Y.; Fujita, H.; Takeuchi, S.; and Noji, H. (2005). Highly coupled ATP synthesis by F1-ATPase single molecules, Nature, 433, pp. 773

[9] Weibel, D. B.; Garstecki, P.; Ryan, D.; DiLuzio, W. R.; Mayer, M.; Seto, J. E. and Whitesides, G. M. (2005). Microoxen: Microorganisms to move microscale loads, Proc. Natl. Acad. Sci. U. S. A., 102, pp. 11963-11967

[10] Neves, H.P., Schmidt, J.J., Soong, R.K., Montemagno, C.D., World Automation Congress, 2002. Proceedings of the 5th Biannual, v.14, pp.275-279 (2002).

[11] Xi, J.; Schmidt J. J. and Montemagno, C. D. (2005). Self-assembled microdevices driven by muscle, Nat. Mater., 4, 180

[12] Tanaka, Y.; Morishima, K.; Shimizu, T.; Kikuchi, A.; Yamato, M.; Okano, T.; and Kitamori, T. (2006). Demonstration of a PDMS-based bio-microactuator using cultured cardiomyocytes to drive, Lab on a chip. 6, pp. 362-368

[13] Fisher, M. E. \& Kolomeisky, A. B. (1999). The force exerted by a molecular motor, Proc. Natl. Acad. Sci. U. S. A., 96, pp. 6597

[14] Entcheva, E. \& H. Bien. (2003). Tension development and nuclear eccentricity in topographically controlled cardiac, Biomed. Microdevices. 5: pp. 163-168,

[15] Motlagh, D.; Hatman, T.; Desai, T \& Russell, B. J (2003).Microfabricated grooves recapitulate neonatal myocyte connexin 43 and $\mathrm{N}$-cadherin, Biomed Mater Res A. 67, pp. $148-57$

[16] Motlagh, D.; Senyo, S. E.; Desai, T and Russell, B. (2003).Microtextured substrata alter gene expression, protein localization and the shape of cardiac, Biomaterials, 24, pp. 2463-2476

[17] Dalby, M. J.; M. O. Riehle; S. J. Yarwood; C. D. W. Wilkinson and A. S. G. Curtis. (2003). Nucleus alignment and cell signaling in fibroblasts: response to a micro-grooved topography, Exper. Cell. Res. 284: pp. 274-282 
[18] Yin, L.; Bien, H. and Entcheva, E. (2004). Scaffold topography alters intracellular calcium dynamics in cultured cardiomyocyte, Am J Physiol Heart Circ Physiol. 287, H pp. 1276-1285

[19] Armani, D.; Liu, C.; Aluru, N. (1999). Re-configurable fluid circuits by PDMS elastomer micromachining, IEEE Micro Electro Mechanical Systems; Orlando, FL, U.S.A. pp 222-227

[20] Park J Y; Ryu J; Choi S K; Seo E; Cha J M; Ryu S; Kim J; Kim B and Lee S H. (2005). Realtime measurement of the contractile forces of self-organized cardiomyocytes on hybrid, Anal. Chem. 77, pp. 6571-6580

[21] Sia, S.; Whitesides, G. (2003). Microfluidic devices fabricated in poly (dimethylsiloxane) for biological studies, Electrophoresis, 24, pp. 3563-3576

[22] B. H. Jo; L. M. Van Lerberghe; K. M. Motsegood and David J. Beebe (2000). Threedimensional micro-channel fabrication in polydimethylsiloxane (PDMS) elastomer, J. Microelectromech. Syst., 9(1), 76-81.

[23] Y. Xia \& G. Whitesides Replica molding with a polysiloxane mold provides this patterned microstructure, Angew. Chem., Int. Ed., 1998, 37, pp. 550-575.

[24] M. Zile, et. al., (1998). Constitutive properties of adult mammalian cardiac muscle cells, Circulation, vol. 98, pp. 567-579

[25] M. Kawai; Y. Saeki; Y. Zhao (1993).Crossbridge scheme and the kinetic constants of elementary steps deduced from chemically, Circulation research: J. American Heart Association, vol. 73, pp. 35-50

[26] K. P. Roos (1997). The Myocardium, 2nd ed. G.A. Langer, editor, Academic, New York. pp. 235-323

[27] G. Lin; R. Palmer; K. Pister, and P. Roos (2000). Surface micromachined polysilicon heart cell force transducer, IEEE Trans. J. Biomedical Engineering, vol. 48, pp. 9961006

[28] Y. Zhao, X. Zhang, IEEE Micro Electro Mechanical Systems, pp. 834-837 (2005).

[29] S. Sia; G. Whitesides (2003). Microfluidic devices fabricated in poly (dimethylsiloxane) for biological studies, Electrophoresis, vol. 24, pp. 3563-3576

[30] Schmidt, J.J.; Montemagno, C.D. (2004). Bionanomechanical systems, Annual Review of Materials Research 34, pp. 315-337.

[31] Janz, R.F.; Grimm, A.F., (1972). Finite-element model for the mechanical behaviour of the left ventricle: prediction of deformation in the potassium-arrested rat heart, Circular Research 30, 244-252.

[32] Park, J.; Kim, J.; Roh, D.; Park, S.; Kim, B.; Chun, K. (2006). Fabrication of 3D thin polymer structures for hybrid sensors and actuators, Journal of Micromechanics and Microengineering 16, 1614-1619. 


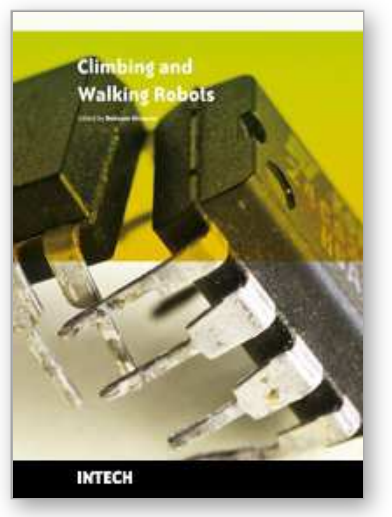

\author{
Climbing and Walking Robots \\ Edited by Behnam Miripour
}

ISBN 978-953-307-030-8

Hard cover, 508 pages

Publisher InTech

Published online 01, March, 2010

Published in print edition March, 2010

Nowadays robotics is one of the most dynamic fields of scientific researches. The shift of robotics researches from manufacturing to services applications is clear. During the last decades interest in studying climbing and walking robots has been increased. This increasing interest has been in many areas that most important ones of them are: mechanics, electronics, medical engineering, cybernetics, controls, and computers. Today's climbing and walking robots are a combination of manipulative, perceptive, communicative, and cognitive abilities and they are capable of performing many tasks in industrial and non- industrial environments. Surveillance, planetary exploration, emergence rescue operations, reconnaissance, petrochemical applications, construction, entertainment, personal services, intervention in severe environments, transportation, medical and etc are some applications from a very diverse application fields of climbing and walking robots. By great progress in this area of robotics it is anticipated that next generation climbing and walking robots will enhance lives and will change the way the human works, thinks and makes decisions. This book presents the state of the art achievments, recent developments, applications and future challenges of climbing and walking robots. These are presented in 24 chapters by authors throughtot the world The book serves as a reference especially for the researchers who are interested in mobile robots. It also is useful for industrial engineers and graduate students in advanced study.

\title{
How to reference
}

In order to correctly reference this scholarly work, feel free to copy and paste the following:

Jinseok Kim, Eui-Sung Yoon and Sukho Park (2010). Biohybrid Walking Microrobot with Self-Assembled Cardiomyocytes, Climbing and Walking Robots, Behnam Miripour (Ed.), ISBN: 978-953-307-030-8, InTech, Available from: http://www.intechopen.com/books/climbing-and-walking-robots/biohybrid-walking-microrobotwith-self-assembled-cardiomyocytes

\section{INTECH}

open science | open minds

\section{InTech Europe}

University Campus STeP Ri

Slavka Krautzeka 83/A

51000 Rijeka, Croatia

Phone: +385 (51) 770447

Fax: +385 (51) 686166

\section{InTech China}

Unit 405, Office Block, Hotel Equatorial Shanghai

No.65, Yan An Road (West), Shanghai, 200040, China 中国上海市延安西路65号上海国际贵都大饭店办公楼 405 单元

Phone: +86-21-62489820

Fax: +86-21-62489821 
www.intechopen.com 
(C) 2010 The Author(s). Licensee IntechOpen. This chapter is distributed under the terms of the Creative Commons Attribution-NonCommercialShareAlike-3.0 License, which permits use, distribution and reproduction for non-commercial purposes, provided the original is properly cited and derivative works building on this content are distributed under the same license. 